\title{
A Neonatal Case with a Prenatal Diagnosis of Capillary Malformation-arteriovenous Malformation Syndrome (CM-AVM)
}

\author{
Somia Errazaki ${ }^{1}$, Martina Marangoni ${ }^{2}$, Guillaume Smits ${ }^{2}$, Boris Lubicz ${ }^{3}$, Dorottya Kelen ${ }^{1}$ \\ ${ }^{1}$ Neonatal Department, Université Libre de Bruxelles, Brussels, Belgium \\ ${ }^{2}$ Genetic Department, Université Libre de Bruxelles, Brussels, Belgium \\ ${ }^{3}$ Interventional-Radiology Department, Université Libre de Bruxelles, Brussels, Belgium
}

Email address:

Soumia.errazaki@ulb.be (S. Errazaki), Martina.marangoni@erasme.ulb.ac.be (M. Marangoni),

Guillaume.smits@erasme.ulb.ac.be (G. Smits), Boris.lubicz@erasme.ulb.ac.be (B. Lubicz), Dorottya.kelen@ erasme.ulb.ac.be (D. Kelen)

\section{To cite this article:}

Somia Errazaki, Martina Marangoni, Guillaume Smits, Boris Lubicz, Dorottya Kelen. A Neonatal Case with a Prenatal Diagnosis of Capillary Malformation-arteriovenous Malformation Syndrome (CM-AVM). American Journal of Pediatrics. Vol. 7, No. 3, 2021 , pp. 145-149.

doi: 10.11648/j.ajp.20210703.20

Received: June 28, 2021; Accepted: July 23, 2021; Published: August 9, 2021

\begin{abstract}
Capillary malformation arteriovenous malformation syndrome (CV-AVM) is marked by the appearance of vascular lesions on the skin, frequently found on the face and limbs. The main genetic defect in this syndrome is a mutation in the RASA-1 gene transmitted in an autosomal dominant manner. Besides, this syndrome is characterized by a wide intra- and interfamilial variability in clinical manifestations. In fact, little is known about prenatally diagnosed cases of CV-AVM. Here, we report the case of a 25 year-old woman admitted during her third pregnancy for management of unexplained polyhydramnios. Prenatal genetic analysis (Mendeliom sequencing from amniotic fluid), performed due to two previous unexplained fetal losses, showed a mutation in a heterozygous state in $R A S A-1$ gene c.2367 C $>\mathrm{T}$ (p.Arg789*). Hence, an antenatal diagnosis of CV AVM was then made. An emergency cesarean section was performed at $315 / 7$ weeks due to complications of the second amnioreduction. At birth, the neonate presented five erythematous lesions with diameters of 1-3 cm, similarly to those found on the father. No signs of arteriovenous malformations was detected on MRI of the brain and spine. No other anomaly has been found. Despite its autosomal mode of transmission, CV-AVM is still underrecognized due to its variable clinical presentation. Our report underlines that CM-AVM should be considered at the first clinical examination of a neonate revealing atypical capillary malformations especially in the context of unexplained polyhydramnios, previous fetal losses, or family history of clinical presentation of CM. When genetic diagnose is made a follow-up should be organized during the period of early childhood.
\end{abstract}

Keywords: Capillary Malformations, Arteriovenous Malformation, Arteriovenous Fistulas, RASA1, EPHB4

\section{Introduction}

Capillary malformation-arteriovenous malformation syndrome (CM-AVM) was first described in 2003 [1]. Cutaneous vascular lesions measuring 1 to $3 \mathrm{~cm}$ in diameter are the hallmark of the disease. These capillary malformations (CM) are frequently found on the face and upper and lower extremities. Furthermore, $<<$ fast-flow $>>$ vascular deformities such as AVM and AVF could be observed among one third of affected individuals and is responsible of significant morbidity over time [2,3]. The main genetic defect in this syndrome is a mutation in the RASA-1 gene involved in angiogenesis. Concerning clinical manifestations, an intra and interfamilial variability has been described. While CM is most commonly seen at birth, AVM and AVF may not appear until later in life $[2,4]$. To our knowledge, published papers on antennal diagnosis of CM-AVM is scarce. It is also well known that pregnancy complications such as polyhydramnios could hide genetic defects [5] particularly if associated with previous miscarriages [6]. In this case, we report a rare prenatal 
diagnosis of RASA-1 gene mutation. Mendeliome (clinical exome) sequencing was performed due to unexplained polyhydramnios and two previous in-utero fetal deaths. It showed a $R A S A-1$ gene mutation.

\section{Case Presentation}

Prenatally, a 25 years old mother was admitted in our institution for management of unexplained polyhydramnios during her third pregnancy. During the second amnioreduction procedure, an emergency cesarean-section was performed at $315 / 7$ weeks due to the onset of contractions and acute fetal distress. Baby boy was born with a birth weight of $1990 \mathrm{~g}$ (P50-P90) and with a head circumference of $29.5 \mathrm{~cm}$ (P50-P90). At birth the patient showed signs of respiratory distress syndrome (RDS), received surfactant followed by non-invasive ventilation for 30 days. Neurologically, on day 1 and 2 of life, the patient presented myoclonic movements of the limbs. The electroencephalogram (EEG) performed showed no seizures with a normal background pattern. The neonate was hemodynamically stable throughout his stay at the unit. Five erythematous lesions between 1-3 cm of diameter were confirmed by physical examination on the chest, right leg and left buttock, left nostril and forehead (Figures 1-2).

The remaining physical examination was normal. No abnormalities were identified with routine blood tests. Prenatal genetic analysis, consisting in a mendeliome (clinical exome) sequencing on amniotic fluid cells showed a mutation in the heterozygous state in the RASA1 gene c.2367 $\mathrm{C}>\mathrm{T}$ (p.Arg789*) resulting in a premature stop codon and a subsequent truncated protein. This same mutation has already been reported by Revencu et al [7].

Moreover, the mutation was also found by the patient's father who showed the similar clinical manifestation of disseminated multiple erythematous lesions.

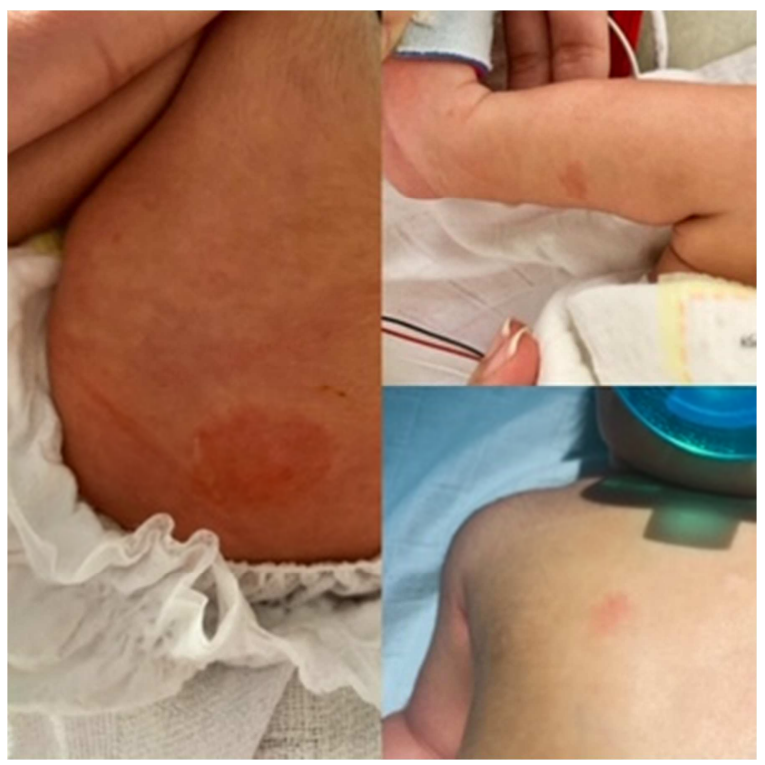

Figure 1. Pink colored macules on the chest, leg and buttock
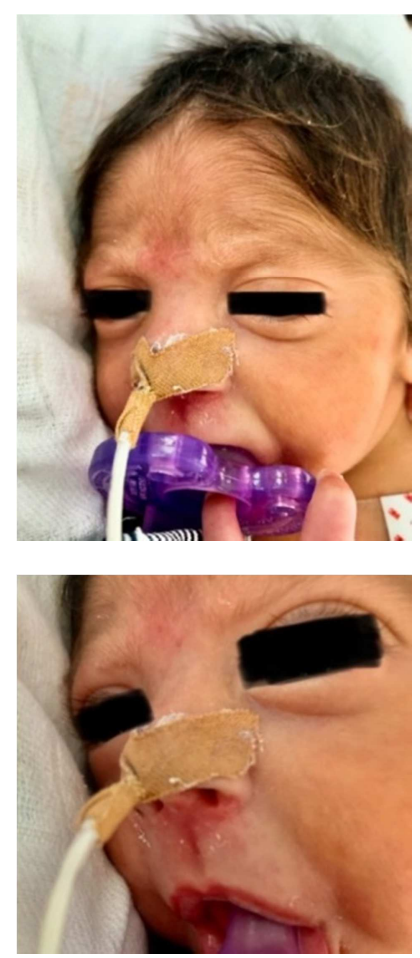

Figure 2. Red lesion on the left nostril and forehead.

The imaging studies performed to detect intracranial or spinal lesions were normal. The reproductive history of the couple is remarkable for two fetal deaths at 12 and at 31 weeks of gestation. The antenatal ultrasounds during the second pregnancy revealed polyhydramnios. Parents refused autopsy in both cases. As for family history of CM or cerebrovascular accident, it turned negative in both parents (Figure 3).

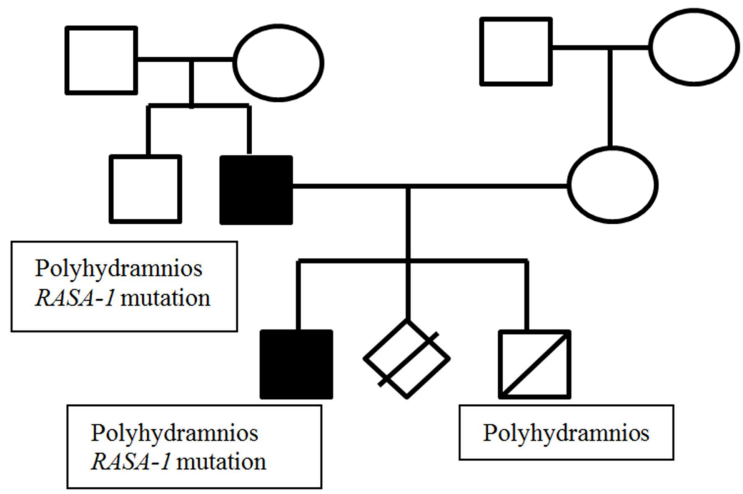

Figure 3. Family tree showing case of RASA-1 mutation

To investigate the extent of the disease, multiple neonatal examinations were performed. We first performed spine and brain MRI which did not show any vascular anomaly. However arteriovenous microfistulas cannot be ruled out at this age. The echocardiography found a small atrial septal defect (type II) without signs of overload. Abdominal ultrasound was normal and the fundoscopic exam showed retinal hypopigmentation related to prematurity without any other vascular abnormalities at this stage. Arterial flow was detected in three out of the five superficial lesions by Doppler ultrasound (Figure 4). 
Finally, the patient was discharged home at 38 weeks of gestation.

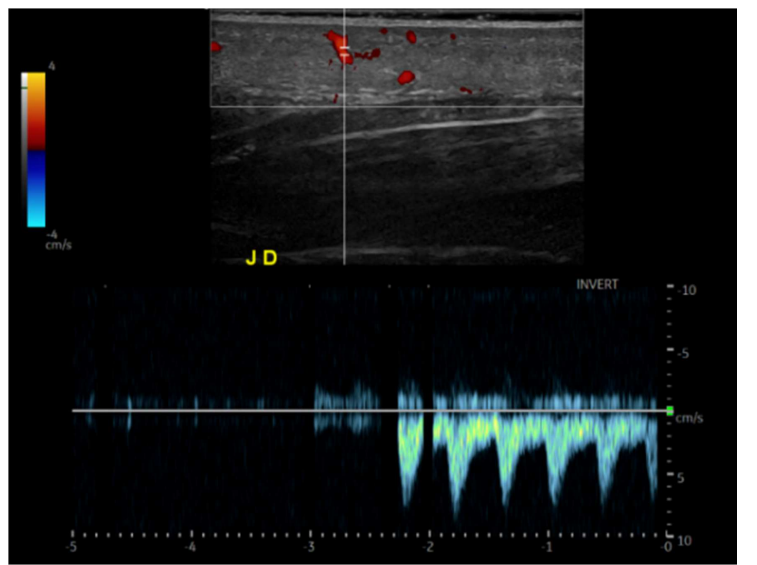

Figure 4. Doppler of the lesion on the right leg showing an arterial flow.

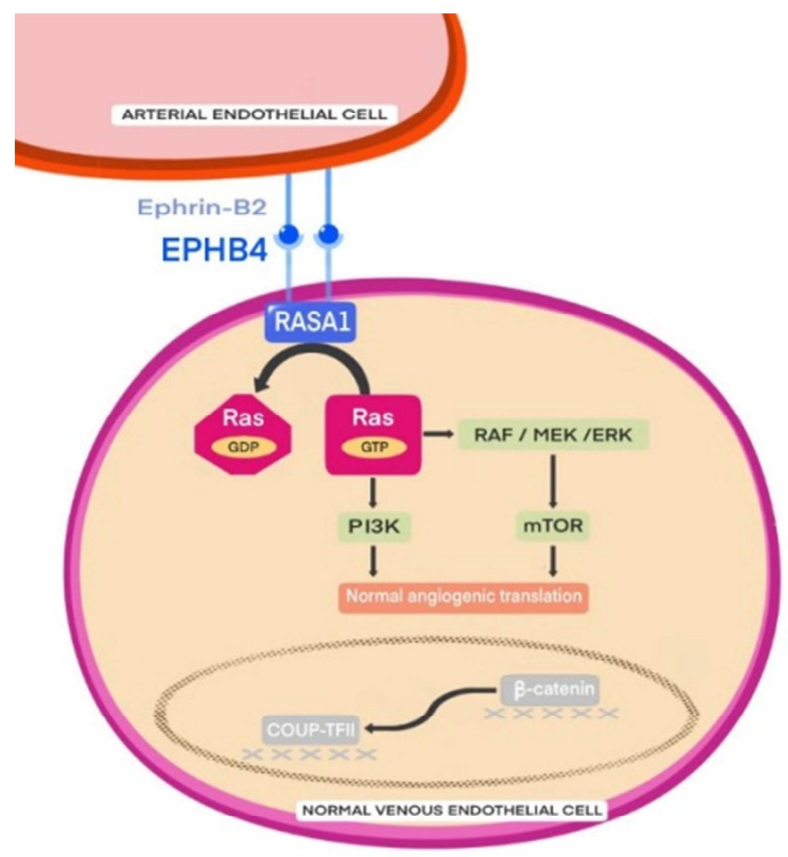

\section{Discussion}

CM-AVM is a rare genetic condition that affects blood vessels. It results from a heterozygous inactivating mutations in the $R A S A-1$ gene (5q13.3) and inherited in an autosomal dominant manner. Nevertheless, de novo mutation is seen in about one third of patients with this conditions, while only two-third have an affected parent $[4,8]$, which is the case of the father in this report. The characteristic findings of this disease, as seen in the child and the father, are erythematous skin lesions arising from angiogenesis anomaly in the protein p120RasGap encoded by $R A S A-1$ gene which is involved in the inhibition of RAS-mediated angiogenesis [9]. This protein regulates as well RAS p21 oncogene [9]. Furthermore, in an experimental study on mouse embryos conducted by $\mathrm{M}$. Henkemyers et al. disrupted rasGap protein led to endothelial cells aggregation in a honeycomb pattern with failure to reorganize into a vascular network [9] (Figure 5).

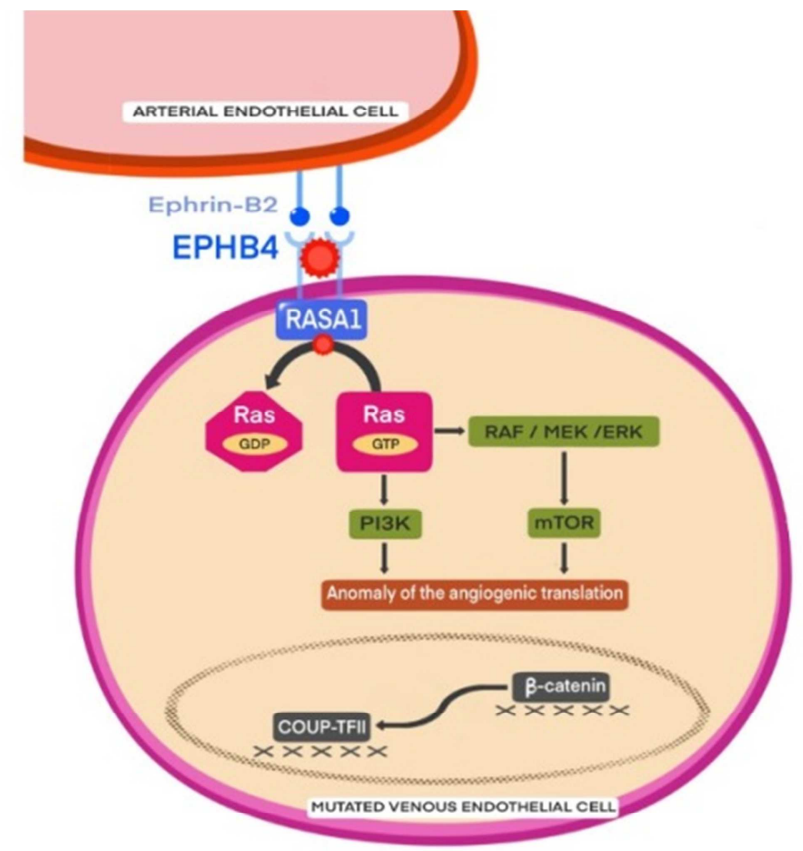

Figure 5. Molecular changes arising from mutations in RASA1 and EPHB4 in a CM-AVM affected endothelial cell. Normally, EPHB4 interact with its ligand EFNB2 (Ephrin-B2 present on endothelial arterial cells and activates RASA1 which inhibits cascades in MAP kinase pathway. Mutations in EPBH4 and RASA-1 result in activation of these cascades and subsequent abnormally differentiate endothelial cells and unorganized vascular network.. Adapted from Gourier G et al) [12].

This disorder has genetic heterogeneity most frequently affecting the RASA-1 gene with more than hundreds different mutations already reported [10] and a high penetrance estimated around 90-99\% [7]. Another possible genetic origin of this condition, previously described by Lacalm et al, is a mutation in EPHB4 encoding a tyrosine kinase receptor that plays a role in angiogenesis [11]. To be distinguished, Amyere et al designated the phenotype arising from RASAl pathological mutations "capillary malformation-arteriovenous malfornmation 1" (CM-AVM1) observed in our patient; and the phenotype deriving from EPHB4 pathological mutations "capillary malformation-arteriovenous malformation 2" (CM-AVM2) [4, 12]. Despite this syndrome description since 2003, it is still underdiagnosed and the prevalence is underestimated. Clinically, it is marked by multifocal and progressive skin lesions between 1 to $3 \mathrm{~cm}$ in diameter. These macules can be pink, brown or red with a white halo in about half of the cases, mostly found on the face and extremities. In some of these lesions, Doppler ultrasound shows arterial flow, which was also affirmative in some of our patient's lesions [7]. Moreover, in a retrospective prospective study conducted by Revencu et al, a new phenotype of CM AVM was reported among several participants described as white halos with central red spots 
that appears on the limbs [8]. Furthermore, a multicentric study conducted in Spain discovered a new large macule, common among CM-AVM patients, and called it Herald patches [13] In addition, some affected individuals present a «fast flow» vascular malformation that includes AVM and AVF [2]. In around 7\% of patients with CM-AVM, we could encounter symptomatic intracranial and spinal fast flow abnormal tangle of blood vessels that frequently occur before 7 years of age [7]. However, it should be noted that AVMs/AVFs may develop over time and cannot be predicted $[9,10]$, hence the importance of a follow-up. Early appearing intracranial AVMs/AVFs present as headaches and migraine or lead to seizures, hydrocephalus and cardiac failure. As for extracranial AVMs/AVFs, they predominantly manifest on the skin, muscle and spine [4, 7]. An intra- and interfamilial variability has been described concerning the number of CMs and the presence of AVMs or AVFs, and this was also evident in our patient's family.

As a reminder, our patient was initially admitted to our hospital for the management of unexplained polyhydramnios. It is interesting to note that the father who carries the same mutation of the RASA-1 gene also presented a polyhydramnios on prenatal ultrasounds. In addition, several cases of neonatal or prenatal presentation of $R A S A-1$ gene mutations or other capillary malformations described in the literature also presented as an unexplained polyhydramnios $[14,15]$. When capillary malformation is suspected, it is of great importance to establish a differential diagnosis that should include: hereditary hemorrhagic telangiectasia, Sturge-Weber syndrome (SWS), Klippel-Trenaunay-Weber syndrome, PTEN hamartoma tumor syndromes, multiple cutaneous and mucosal venous malformations and hereditary glomuvenous malformations (GVMs) [4, 7]. Diagnostic criteria for CM AVM1 syndrome caused by RASA-1 mutations have been described in the literature $[9,16]$ (Table 1).

Based on the criteria by Orme et Weitz, our patient has a definitive diagnosis of CM-AVM syndrome [9, 16]. However, there are no established guidelines concerning the assessment, management and follow-up of these patients. In our clinical case we proposed the following plan:

1) Angio-MRI for screening of intracranial and spinal AVMs/AVFs in three years' time, or earlier if the patient develops symptoms.

2) Genetic counseling to help the family to better understand the risks associated with this pathology.

3) The possibility of prenatal or preimplantation genetic testing in case of future pregnancies.

Table 1. Proposed Diagnostic Criteria and Evaluation Guidelines for CM-AVM.

\begin{tabular}{ll}
\hline Proposed diagnostic criteria for CM-AVM & \\
\hline Definite CM-AVM & Probable CM-AVM \\
\hline & $>3$ characteristic CMs \\
Numerous characteristic CMs & or \\
+/-AVMs & Characteristic CMs + AVM \\
+/-Family history of CMs & or \\
(+/-AVM) & Characteristic \\
+/-Confirmed RASA-1 mutations & CMs + family \\
& history of \\
\hline
\end{tabular}

CM-AVM, capillary malformation-arteriovenous malformation syndrome; CNS, central nervous system. Adapted from Orme et al [9] et Weitz et al [15]

\section{Conclusion}

CM-AVM is not a well-known condition and could be underdiagnosed. We have observed that polyhydraminos is often reported in cases of CM-AVM caused by RASAl gene mutations, and could be a useful element of the diagnosis. Further research is needed to investigate this observation. Initial presentation of our patient only revealed capillary malformations, nevertheless there is a possibility that AVMs/AVFs develop later in life given the intra-familial phenotypic variability, implying the need of clinical and imaging follow-up.

\section{References}

[1] Eerola I, Boon LM, Mulliken JB, Burrows PE, Dompmartin A, Watanabe $\mathrm{S}$ et al. Capillary Malformation-Arteriovenous Malformation, a New Clinical and Genetic Disorder Caused by RASA1 Mutations. Am J Hum Genet. 2003 Dec; 73 (6): $1240-9$.
[2] Cai R, Liu F, Liu Y, Chen H LX. RASA-1 somatic "second hit" mutation in capillary malformation-arteriovenous malformation. J Dermatol. 2018 Dec; 45 (12): 1478-1480.

[3] Behr GG, Liberman L, Compton J, Garzon MC, Morel KD, Lauren CT et al. CM-AVM syndrome in a neonate: case report and treatment with a novel flow reduction strategy. Vasc Cell. 2012; 4 (1): 19.

[4] Bayrak-Toydemir $\quad P \quad$ SD. Capillary Malformation-Arteriovenous Malformation Syndrome. In: Adam MP, Ardinger HH, Pagon RA, Wallace SE, Bean LJ, Mirzaa G, et al., editors. GeneReviews ${ }^{\circledR}$ [Internet]. Seattle (WA): University of Washington, Seattle; 1993. Available from: http://www.ncbi.

[5] Boito S, Crovetto F, Ischia B, Crippa BL, Fabietti I, Bedeschi MF et al. Prenatal ultrasound factors and genetic disorders in pregnancies complicated by polyhydramnios. Prenat Diagn. 2016 Aug; 36 (8): 726-30.

[6] Tur-Torres MH, Garrido-Gimenez C A-RJ. Genetics of recurrent miscarriage and fetal loss. Best Pract Res Clin Obstet Gynaecol. 2017 Jul; 42: 11-25. 
[7] Revencu N, Boon LM, Mulliken JB, Enjolras O, Cordisco MR, Burrows PE et al. Parkes Weber syndrome, vein of Galen aneurysmal malformation, and other fast-flow vascular anomalies are caused by RASA1 mutations. Hum Mutat. 2008 Jul 1; 29 (7): 959-65.

[8] Orme CM, Boyden LM, Choate KA, Antaya RJ KB. Capillary Malformation-Arteriovenous Malformation Syndrome: Review of the Literature, Proposed Diagnostic Criteria, and Recommendations for Management. Pediatr Dermatol. 2013 Jul 1; 30 (4): 409-15.

[9] Henkemeyer M, Rossi DJ, Holmyard DP, Puri MC, Mbamalu G Harpal K et al. Vascular system defects and neuronal apoptosis in mice lacking Ras GTPase-activating protein. Nature. 1995 Oct; 377 (6551): 695-701.

[10] Gourier G, Audebert-Bellanger S, Vourc'h P, Fraitag S, L'Hérondelle K, Labouche A et al. Multiple capillary malformations of progressive onset: Capillary malformationarteriovenous malformation syndrome (CM-AVM). Ann Dermatol Vénéréologie. 2018 Aug; 145 (8-9): 486-91.

[11] Lacalm A, Fichez A, Broussin B, Abel C, Lacombe D GL. Prenatal diagnosis of cerebral and extracerebral high-flow lesions revealing familial capillary malformation-arteriovenous malformation (CM-AVM) syndrome. Ultrasound Obstet Gynecol. 2018 Mar 1; 51 (3): 409-11.
[12] Amyere Mustapha, Revencu Nicole, Helaers Raphaël, Pairet Eleonore, Baselga Eulalia, Cordisco Maria et al. Germline Loss-of-Function Mutations in EPHB4 Cause a Second Form of Capillary Malformation-Arteriovenous Malformation (CM-AVM2) Deregulating RAS-MAPK Signaling. Circulation. 2017 Sep 12; 136 (11): 1037-48.

[13] Valdivielso-Ramos M, Martin-Santiago A, Azaña JM, Hernández-Nuñez A, Vera A PB. Capillary malformation-arteriovenous malformation syndrome: a multicentre study. Clin Exp Dermatol. 2021 Mar; 46 (2): 300-305.

[14] Revencu N, Boon LM, Mendola A, Cordisco MR, Dubois J, Clapuyt $\mathrm{P}$ et al. RASA1 mutations and associated phenotypes in 68 families with capillary malformation-arteriovenous malformation. Hum Mutat. 2013 Dec; 34 (12): 1632-41.

[15] Coste K, Sarret C, Cisse A, Delabaere A, Francannet C VP. Le syndrome macrocéphalie - malformation capillaire. Intérêt du diagnostic en période néonatale. Arch Pédiatrie. 2012 Sep; 19 (9): 917-20.

[16] Weitz NA, Lauren CT, Behr GG, Wu JK, Kandel JJ, Meyers PM et al. Clinical Spectrum of Capillary MalformationArteriovenous Malformation Syndrome Presenting to a Pediatric Dermatology Practice: A Retrospective Study. Pediatr Dermatol. 2015 Jan 1; 32 (1): 76-84. 